\title{
Open-Source Potentiostat for Wireless Electrochemical Detection with Smartphones
}

\author{
Alar Ainla, ${ }^{\dagger, \dagger, \#}$ Maral P. S. Mousavi, ${ }^{\dagger, \#}$ Maria-Nefeli Tsaloglou, ${ }^{\dagger, \S}$ Julia Redston, ${ }^{\dagger}$ Jeffrey G. Bell, ${ }^{\dagger}$

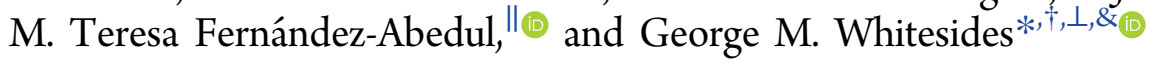

${ }^{\dagger}$ Department of Chemistry and Chemical Biology, ${ }^{\perp}$ Wyss Institute for Biologically Inspired Engineering, and ${ }^{\&}$ Kavli Institute for Bionano Science and Technology, Harvard University, Cambridge, Massachusetts 02138, United States

${ }^{\ddagger}$ International Iberian Nanotechnology Laboratory (INL), Braga 4715-330, Portugal

${ }^{\S}$ Diagnostics for All, Inc. (DFA), Salem, Massachusetts 01970, United States

"Department of Physical and Analytical Chemistry, University of Oviedo, Oviedo 33003, Spain

\section{Supporting Information}

ABSTRACT: This paper describes the design and characterization of an open-source "universal wireless electrochemical detector" (UWED). This detector interfaces with a smartphone (or a tablet) using "Bluetooth Low Energy" protocol; the smartphone provides (i) a user interface for receiving the experimental parameters from the user and visualizing the result in real time, and (ii) a proxy for storing, processing, and transmitting the data and experimental protocols. This approach simplifies the design, and decreases both the size and the cost of the hardware; it also makes the UWED adaptable to different types of analyses by simple modification of the software. The UWED can perform the most common electroanalytical techniques of potentiometry, chronoamperome-

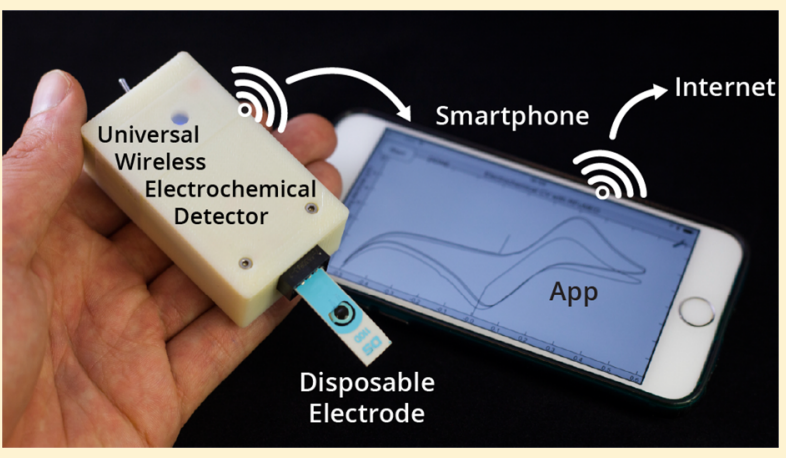
try, cyclic voltammetry, and square wave voltammetry, with results closely comparable to benchtop commercial potentiostats. Although the operating ranges of electrical current and voltage of the UWED $( \pm 1.5 \mathrm{~V}, \pm 180 \mu \mathrm{A})$ are more limited than most benchtop commercial potentiostats, its functional range is sufficient for most electrochemical analyses in aqueous solutions. Because the UWED is simple, small in size, assembled from inexpensive components, and completely wireless, it offers new opportunities for the development of affordable diagnostics, sensors, and wearable devices.

$\mathrm{E}$ lectrochemistry is useful for analyses in applications including clinical diagnostics, environmental, industrial, and food monitoring, quality control, and wearable devices for personal health. ${ }^{1-8}$ Different techniques have enabled the electrochemical detection of analytes including proteins, DNA, ions, neurotransmitters, and other small electroactive molecules. $^{1-12}$ A potentiostat-an instrument with electronic circuits for the control and measurement of the electrical current and voltage-is necessary for performing the electroanalysis. Advances in electronics have enabled simplifying the large, heavy, and expensive benchtop potentiostats to portable and low-cost electric readers that are suitable for analysis at the point-of-use and in resource-limited or field settings.

With appropriate technical competence, fabrication of portable potentiostats is not a scientific challenge. In fact, portable potentiostats that can perform most electrochemical techniques are now commercially available at an approximate cost of a few thousand dollars. ${ }^{13-15}$ This cost still prohibits their widespread use, specifically in resource-limited applications. Not surprisingly, the components and circuit design of the commercial potentiostats are proprietary and inaccessible to those who need to develop an affordable potentiostat for research, education, and product integration. Simpler readers designed for a specific application are available at a much lower price $(<\$ 100)$. Commercial glucometers are a widely available example. Such readers can be repurposed for other electroanalytical tests, but there are many limitations to the capabilities of these instruments (e.g., the range and resolution of electrical current and voltage, and type of electroanalytical techniques that they can perform). ${ }^{16,17}$ The potential value of open-source, portable, electrical readers for point-of-care diagnostics is wellrecognized. There are an increasing number of publications that are entirely or partly dedicated to describing the development of such potentiostats. ${ }^{18-35}$ Technology developed in an opensource format allows researchers to adopt the design of the device easily, and to develop specific-purpose potentiostats without the need to design and test the electronics from scratch.

Received: February 22, 2018

Accepted: April 16, 2018

Published: April 16, 2018 
Description of a truly open-source potentiostat should include all details required to replicate the system, including the following: (i) the design of the electric circuit, including a complete list of components; (ii) the layout of the printed circuit board (PCB); (iii) the firmware of the microcontroller; and (iv) the codes for the computer software, mobile application, and/or server software. To our knowledge, there have been only three publications describing truly open-source portable potentiostats: (i) CheapStat developed by Rowe et al., ${ }^{19}$ (ii) Dstat developed by Dryden et al., ${ }^{18}$ and (iii) a potentiostat/galvanostat for characterization of thin-film batteries by Dobbelaere et al. ${ }^{35}$ They provided a valuable resource for developing an in-house built potentiostat. All three potentiostats are universal, meaning that they can change the operation parameters on-demand (without physical modifications) and can perform most commonly used electrochemical techniques, such as cyclic voltammetry (CV) and chronoamperometry (CA).

The previously published open-source potentiostats ${ }^{18,19,35}$ were designed to be used in conjunction with a computer via a wired connection to the USB port, and did not offer connection to mobile phones. The emergence and unprecedented spread of mobile phones has provided unique opportunities for their use in the medical field. More than $62 \%$ of the global population already owns a mobile phone; and more than $50 \%$ of this population own a smartphone that has advanced computing power and connectivity. ${ }^{36,37}$ A portable potentiostat with wireless connectivity to smartphones would facilitate electrochemical analysis at the point-of-use and in the field, where access to a computer or wired connection to a device is difficult or impossible. This work describes the design and characterization of a portable "universal wireless electrochemical detector", which we refer to as UWED. The UWED is powered by a rechargeable battery, interfaces with a smartphone (or a tablet) through the "Bluetooth Low Energy" (BLE) protocol, and uses the phone both as the user interface and as the proxy for online data storage (e.g., the "Cloud").

There has been prior work on development of wireless smartphone-connected electrochemical detectors for a specific application or technique, but they cannot be categorized as universal or open-source potentiostats. ${ }^{21-24,33}$ To our knowledge, UWED (this work) is the first open-source wireless universal potentiostat that can interface with smartphones and tablets. To fabricate UWED, we combined the analog circuitry of our previously reported portable potentiostat (the universal mobile electrochemical detector or $\mathrm{UMED}^{20,38}$ ) with a BLE microcontroller. We had designed UMED to be a batterypowered, handheld device, with built-in processing capabilities, for application in resource-limited areas where access to computers or high-end electronic devices with data-processing power is limited. ${ }^{20}$ The UMED interfaced with mobile phones for transmitting data to the Cloud. The interfacing was accomplished through the audio jack to ensure compatibility with all mobile devices, ranging from low-end phones to smartphones. $^{20}$

The main limitation of UMED is that the user can employ only a predetermined protocol loaded on the device, and then transmit the final result of the experiment to the Cloud after the end of the experiment. This follow-up work (UWED) provides a portable potentiostat complementary to UMED, where the experimental protocol (controlled by a smartphone or tablet) can be changed simply by the user without the need for altering the hardware or even the firmware of the device. The UWED also enables real-time visualization and complete transmission of the results of the measurement; this feature is helpful for troubleshooting and quality control. We show the application of UWED in four commonly used electrochemical techniques-potentiometry, chronoamperometry (CA), cyclic voltammetry $(\mathrm{CV})$, and square wave voltammetry (SWV) - and demonstrate that performance of our electrochemical reader is comparable to a commercial benchtop potentiostat.

\section{EXPERIMENTAL SECTION}

We used a commercial screen-printed three-electrode cell (DRP-110CNT-GNP, DropSens, Llanera, Spain) for performing $\mathrm{CA}, \mathrm{SWV}$, and $\mathrm{CV}$ on $10 \mu \mathrm{M}-10 \mathrm{mM}$ ferricyanide. We tested the potentiometric mode of UWED using in-house-built $\mathrm{K}^{+}$and $\mathrm{Na}^{+}$ion-selective electrodes (ISEs), which we fabricated according to protocols established in the literature. ${ }^{39}$ Supporting Information lists the details of solution preparation and electrochemical measurements.

The fabrication of the UWED occurred as follows: Using the free version of $\mathrm{CAD}$ software Eagle (version 7.6), we designed the UWED in two steps: (i) we first designed the circuit diagram (Figure S1), and (ii) we then designed the route of the wires and layout of the components on a two-layer printed circuit board (PCB). Figure S2 shows the design and layout of the PCB. We verified the design of the PCB using design rule check and then fabricated it using a commercial custom PCB service (Silver Circuits Sdn. Bhd., Selangor, Malaysia). We ordered all other circuit components from Digi-Key Electronics (Thief River Falls, MN); Table S3 lists the details of the circuit components. We surface-mounted the components on the PCB and manually soldered them to the PCB. To ensure the board does not have any false connections, we inspected it under a microscope and tested the connections with a multimeter.

We wrote the firmware of the microcontroller (RFduino) in the Arduino software v1.6.5 with the RFduino board package v2.3.1. To load the firmware onto the microcontroller, we attached temporary wires to the contact pads on the PCB, and connected the other end to the terminals of a USB shield for RFduino (RFD22121). We removed the temporary wires after loading the firmware. We then soldered the socket to connect to the commercial ceramic card with screen-printed electrode, and the Li-polymer battery ( $\mathrm{LiPo}$ ) to the contact pads. We designed the housing for the UWED in AutoCAD 2013, exported the file as an STL file, and 3D-printed the case in acrylonitrile butadiene styrene (ABS) using a $3 \mathrm{D}$ printer (StrataSys Fortus 250mc). Figure S3 shows the housing and components of UWED before assembly. After assembly, we charged the internal battery with an external LiPo charger (connected to the charging port of UWED). We developed the software for the smartphone and tablet in techBASIC 3.3.3 (Byte Works Inc.) and tested the software on an iPhone 6S and iPad Mini (Apple). The software saves the data in the text format and sends it to the Cloud (for storage and further processing) over e-mail protocol. We analyzed the results of the electrochemical measurements in MatLAB R2014b.

\section{RESULTS AND DISCUSSION}

Table S1 compares and summarizes the features of UWED (this work), UMED (our previously published low-cost electrochemical reader $^{20}$ ), and the three previously published open-source potentiostats. ${ }^{18,19,35}$ The UWED is the only potentiostat that enables wireless electrochemical detection 
and wireless connection to a smartphone or tablet. The wireless detection allows a physical separation between where the potentiostat is controlled and data is interpreted, and where the experiment is performed. A wireless electrical reader has the following advantages over devices that require wired connection to the processing device (computer, phone, or tablet): (i) The device can be used inside closed containers such as desiccators and glovebags/gloveboxes, and biologically isolated units (which might be contaminated with pathogens and toxins, or radioactive materials), and controlled outside of these units. This separation minimizes the risk of cross-contamination and ensures the safety of the user. (ii) The device can be embedded in wearable sensors and can be used in the field (for environmental monitoring and point-of-care diagnostics), without an application-limiting wired connection. (iii) The device is not prone to electronic noise caused by ground loops that occur through a wired connection to the power source.

Design of the UWED. Figure 1A,C shows an illustration and picture of how UWED can be utilized. The device connects to a smartphone or tablet via BLE. A host program in the smartphone or tablet communicates with the user to identify the type of electrochemical technique, and experimental parameters, and sends commands to the UWED to perform the appropriate voltage and current pulses and measurements. The UWED acquires the individual data and sends them in real time to the smartphone. The smartphone collects and aggregates the data, visualizes and processes them, and sends the data further to the Cloud for storage. Interfacing the UWED to a smartphone or tablet has the following benefits: (i) Smartphones and tablets have high computing powers and long battery lives. The processing and computation of the raw results of the experiment can be outsourced from the electrochemical reader to the smartphone or tablet, and can thus increase the capabilities of the analysis without increasing the complexity and price of this reader. (ii) The smartphones and tablets provide an excellent user interface to receive the input parameters from the user and to visualize the data. The elements of the user interface-buttons, display, and indicators-can be eliminated from the electric reader, and thus simplify its design, and decrease its cost and size. (iii) More than $35 \%$ of the global population are currently using smartphones, and this number is expected to rise. ${ }^{37}$ Most users can operate the UWED with the device that they currently own, by simply installing the appropriate software (or "App"). Use of Apps on smartphones and tablets is an operation growing more familiar to most users. Updating the App enables simple upgrading of the capabilities of the analysis. (iv) The UWED transmits the results of electrochemical analyses to the host program in a smartphone or tablet. Most smartphones and tablets are already connected to the Internet via $\mathrm{Wi}-\mathrm{Fi}$ or through $3 \mathrm{G}$ or $4 \mathrm{G}$ networks and, therefore, can immediately transmit the results to the Cloud. Cloud connectivity makes it possible to decouple the archiving and analyzing of information from the site of testing, minimizes user's error in manual entering of the results, allows encrypted data transmission (and thus increases the data security), and enables incorporation of data in a central data-processing system (i.e., in hospitals and clinics).

Figure 1B shows a block diagram of components of UWED. The device integrates these components: (i) a BLE microcontroller with built-in analog-to-digital converter (ADC), (ii) a two-channel digital-to-analog converter (DAC), (iii) a filter, (iv) a three-electrode potentiostat, (v) socket to interface with
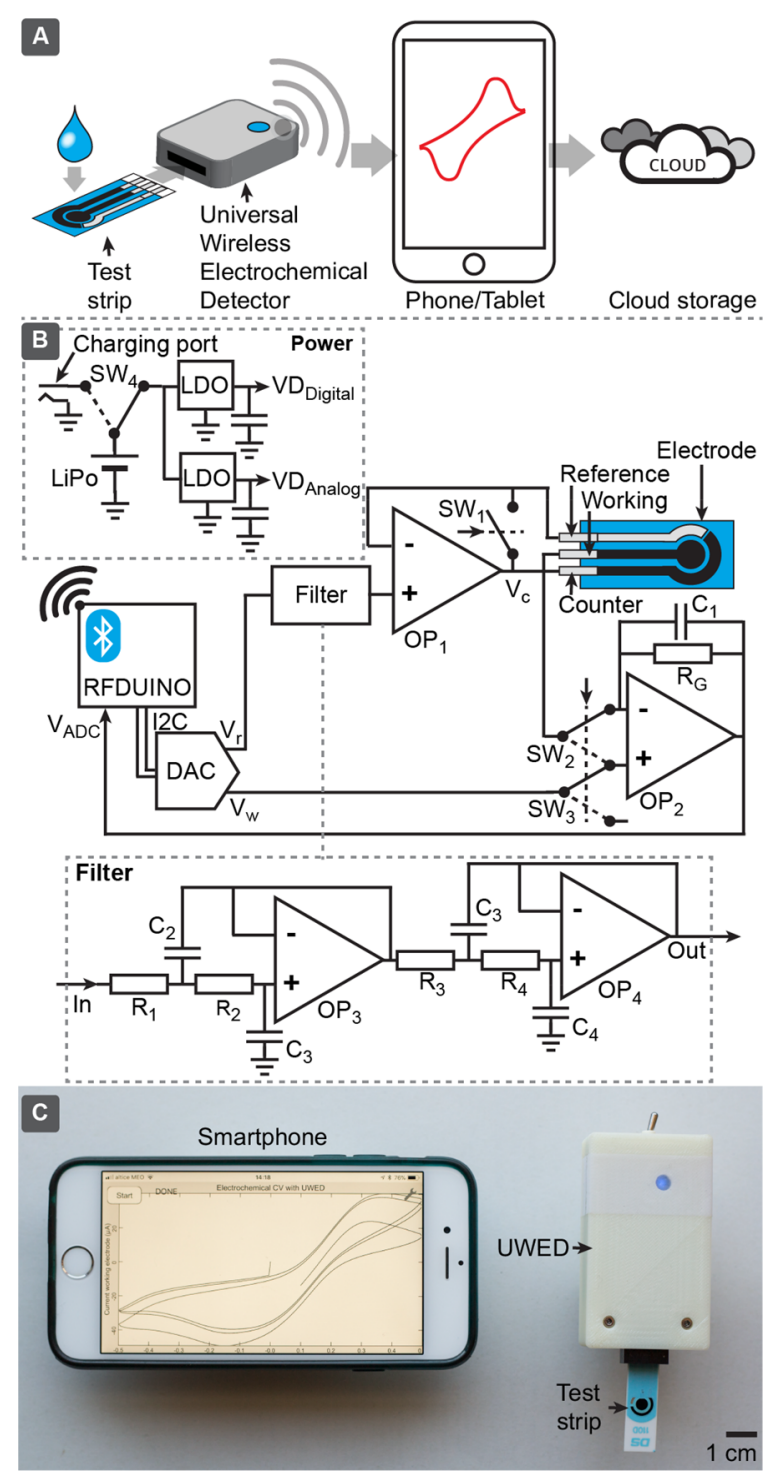

Figure 1. Panels (A) and (C) show how the UWED functions. The working, reference, and counter electrodes are plugged into the UWED which is connected to a smartphone via BLE. A host program in the smartphone controls the experimental protocol, processes, visualizes, and stores the data, and transmits it to the Cloud. (B) shows the circuit diagram and main components of UWED.

screen-printed and other types of electrodes, and (vi) a rechargeable $3.7 \mathrm{~V}$ lithium ion polymer battery for supplying the power to the device.

Microcontroller. Microcontrollers are single chips that contain all the components required for a computing system internally. Most microcontrollers can be programmed by the user for specific functions (the program is referred to as the firmware). In UWED, we used an RFduino microcontroller (ca. $\$ 16 /$ unit), which contains a 32-bit ARM processor, memory, and peripheral components, such as BLE for communication, $\mathrm{I}^{2} \mathrm{C}$ bus for interfacing with DAC, digital outputs for switches, and a 10-bit $\mathrm{ADC}$ with conversion time of $70 \mu \mathrm{s}$ for the measurement of the input signals.

We have RFduino as the BLE microprocessor because its size and shape allow convenient manual soldering; RFduino has several programmable channels for input and output, and is also compatible with the popular open-source Arduino program- 
ming software for convenient development of the firmware. Compatibility with Arduino makes reprogramming of the device simple (to add new electrochemical techniques or alter the existing ones) without the need for development of advanced software. Table S4 lists alternative options for the microcontroller. These options have lower costs or sizes, but are either not suitable for manual soldering or are not compatible with Arduino programming for development of the firmware. We equipped the RFduino microcontroller with firmware that listens to, interprets, and executes the commands sent by the phone or tablet. The commands allow the following functions to be performed: (i) Set the configuration of the potentiostat (two- or three-electrode mode, potential of the electrodes, and application or measurement of voltage or current). (ii) Perform a voltage scan in a number of $(N)$ steps. Each step has a time interval of $\Delta t$, and voltage difference of $\Delta V$ with the preceding step. Section 4 of the Supporting Information provides more details on the development of the firmware.

We have specifically chosen BLE for wireless communication with the external device for four reasons: (i) BLE microprocessors are low power and low cost. (ii) BLE is implemented in all modern smartphones as a common communication mode. UWED is thus compatible with all devices without the need for specialized hardware. (iii) BLE has a much larger working distance (tens of meters) than alterative protocols such as "Near Field Communication" (NFC, working distance of tens of centimeters). (iv) BLE offers a broad bandwidth for data transmission, and thus enables transmitting of all raw data to the smartphone or tablet over the working distance of BLE.

DAC, Filter, and the Three-Electrode Potentiostat. We adopted the design of the analog circuitry from our previous portable electrical reader, UMED. The DAC receives the digital input from the microcontroller (over the $\mathrm{I}^{2} \mathrm{C}$ bus) and converts it into two analog output signals (or voltages), to set the potentials of the reference electrode and working electrode. Section 6 of the Supporting Information lists the details of calibration of DAC. The filter consists of two operational amplifiers and is designed to decrease the noise in the electrical potential. The potentiostat part of the circuit consists of two operational amplifiers (OP1-2), three digital switches (SW13 ), and a gain resistor (RG). The output amplifier, OP1, adjusts the potential of the counter electrode, and feeds back this potential to the reference electrode to ensure that no inconsistencies between the potential of the reference electrode and output of the reference potential in the DAC occur. The digital switches reconfigure the potentiostat between amperometric or potentiometric modes, and between two-electrode or three-electrode modes. The exact function of OP2 depends on the electrochemical technique performed by the UWED. In the potentiometric mode, the microprocessor closes the SW1, the potentiostat is configured to the two-electrode mode, the input impedance is set to high values (G $\Omega$ ), and the output of OP2 corresponds to the potential of the working electrode. In the amperometric mode, feedback is configured (by adjusting the on/off state of SW2 and SW3) to maintain a constant potential for the working electrode, while the current through electrode changes.

Socket to Interface with Electrodes. Use of commercial screen-printed three-electrode cells is growing in popularity because they (i) require small sample volumes (30-200 $\mu \mathrm{L})$, (ii) have relatively low costs $(<\$ 10$ per cell), (iii) enable a variety of materials and surface modifications for the working electrode, thus enabling different types of analyses, and (iv) offer single use and can be disposed of by incineration in biological analyses, where contamination is an issue. Most brands of these commercial screen-printed electrodes (with DropSens as the most recognizable one) have similar designs and dimensions. We equipped UWED with a socket that interfaces with these screen-printed electrodes, and the commercial electrode can be plugged into the socket to perform the electroanalytical measurement. Other types of electrodes can be connected to UWED with an adaptor that has on one end a pin-type connector (to plug in the socket), and the appropriate type of connector on the other end. UWED can be customized by soldering the appropriate connector to the PCB.

Battery. We used a $3.7 \mathrm{~V}$ lithium ion polymer battery for supplying the power to UWED. We set the electronics to operate at $3.3 \mathrm{~V}$ and performed the electrical characterization over $\pm 1.5 \mathrm{~V}$. The battery lasts $40-60 \mathrm{~h}$ if the UWED is actively used for data collection and transmission. Table S8 lists the details of power and current consumption of UWED during different operations. The battery can be recharged through the power outlet or connection to a computer via the USB port. Different batteries have lower weight or longer working time to meet specific demands (the Supporting Information provides a list of examples).

The operational current and voltage window and noise and resolution in voltage and current in UWED is comparable to those of UMED, DStat, and CheapStat (Table S1). Table S9 compares the parameters of UWED to a benchtop commercial potentiostat. The benchtop potentiostats offer an operational window of current and voltage $( \pm 10 \mathrm{~V}, \pm 2 \mathrm{~A})$ wider than that of UWED $( \pm 1.5 \mathrm{~V}, \pm 180 \mu \mathrm{A})$, and also have higher resolution and less noise than UWED. The operational parameters of UWED, however, are sufficient for most commonly used electroanalytical applications. The noise can be decreased by averaging the signal, or by changing the analog circuit and using an ADC with higher resolution. The total cost of all the components for fabrication of one unit of UWED was $\$ 61.50$. We calculated this cost for ordering one unit of components from the supplier. Ordering the components in larger scales (thousands) decreases the cost of one unit of UWED by $75 \%$ to $\$ 15.00$ (The Supporting Information lists the prices of components for large-scale orders).

Electrochemical Comparisons and Demonstrations. We show application of UWED in four commonly used electrochemical techniques of (i) potentiometry, (ii) chronoamperometry, (iii) cyclic voltammetry, and (iv) square wave voltammetry.

Potentiometry. We tested two $\mathrm{K}^{+}$and $\mathrm{Na}^{+}$ISEs using UWED and a commercial potentiometer designed with highinput impedance (EMF 16, Lawson Laboratories Inc.) for potentiometric measurements. Both ISEs exhibited slopes (electrical potential vs logarithm of activity of target ion) close to the theoretically expected Nernstian slope of $59.2 \mathrm{mV} /$ decade. Figure 2A and Figure S13 show good agreement $(<1.5$ $\mathrm{mV}$ difference) between values measured by the UWED and the commercial device. Figures S10 and S11 show traces of potentials of $\mathrm{K}^{+}$and $\mathrm{Na}^{+}$ISEs over time. The UWED appears to have higher noise than the commercial device. The higher noise level in the measurement done with UWED (100-200 $\mu \mathrm{V})$ is due to shorter integration time $(20 \mathrm{~Hz})$ in UWED compared to that in the commercial device $(10-40 \mu \mathrm{V}$ noise, 1 

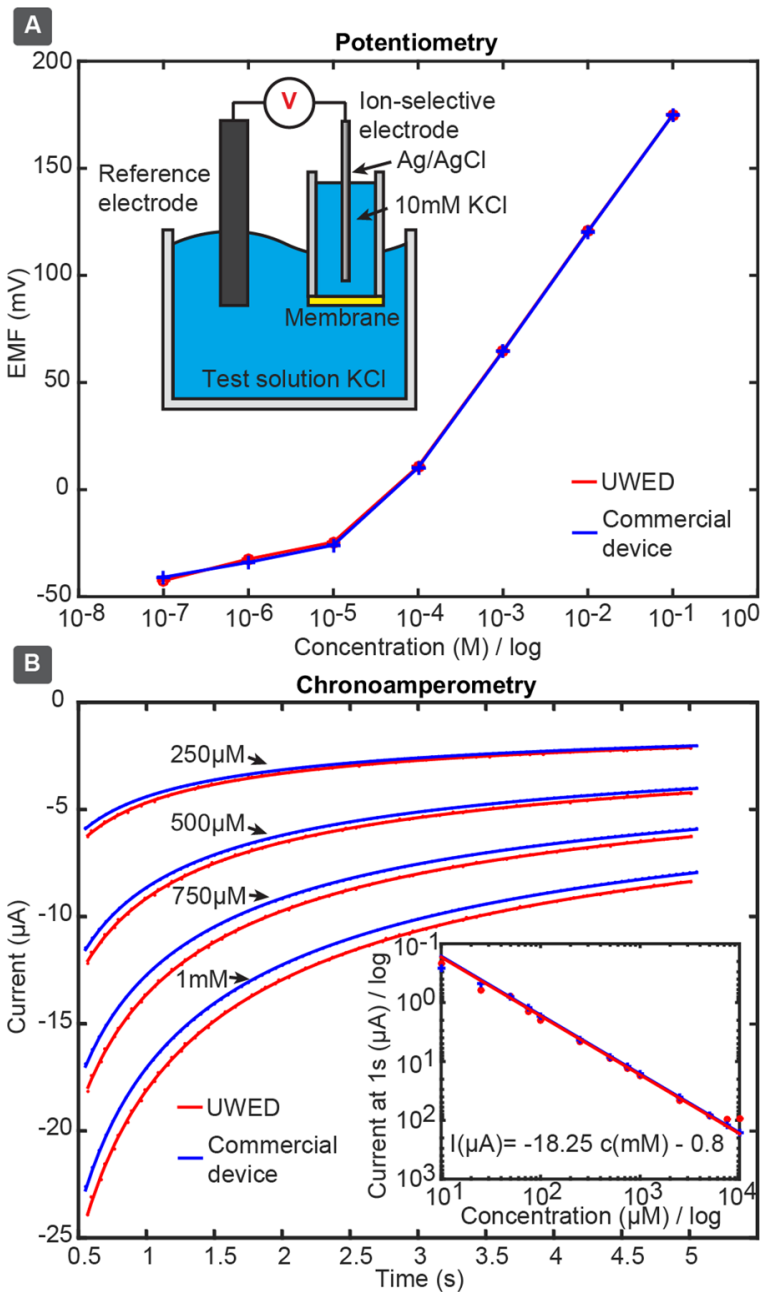

Figure 2. Comparison between the performance of UWED and a commercial benchtop potentiostat [(A) EMF 16, Lawson Laboratories Inc.; (B) Autolab, PGSTAT12], in techniques of potentiometry and chronoamperometry. (A) Potentiometric measurement of the concentration of $\mathrm{K}^{+}$using an ion-selective electrode and a commercial reference electrode. (B) Chronoamperometric measurement of potassium ferricyanide $(1 \mathrm{mM}-250 \mu \mathrm{M})$ on a screen-printed electrode. The inset shows the theoretically expected linear correlation of current (sampled at $1.0 \mathrm{~s}$ ) and concentration of ferricyanide.

$\mathrm{Hz}$ integration time). By increasing the integration time to the same value as the commercial device, we obtained a noise of 20-47 $\mu \mathrm{V}$, which is comparable to that of the commercial device.

Chronoamperometry. We measured chronoamperograms of ferricyanide at concentrations ranging from $25 \mu \mathrm{M}$ to 10 $\mathrm{mM}$. There is excellent agreement between data obtained by the UWED and the commercial potentiostat at concentrations of $25 \mu \mathrm{M}-2.5 \mathrm{mM}$ ferricyanide (Figure $2 \mathrm{~B}$ ). At concentrations of 5-10 $\mathrm{mM}$ the current exceeded the current range of UWED $( \pm 180 \mu \mathrm{A})$, and caused deviation between the measurements of UWED and the commercial potentiostat (Autolab, PGSTAT12, Metrohm). Section 7.2 of the Supporting Information contains all the chronoamperograms. The inset in Figure 2B demonstrates the theoretically expected linear relationship between the current (sampled at $1 \mathrm{~s}$ ) and concentration of ferricyanide.

Cyclic Voltammetry. Cyclic voltammograms (CVs) of ferricyanide at three different concentrations 10.0, $0.1 \mathrm{mM}$ (scan rate of $100 \mathrm{mV} / \mathrm{s}$ ), and $1.0 \mathrm{mM}$ (scan rates of $20-300$ $\mathrm{mV} / \mathrm{s}$ ) recorded by the UWED and the commercial potentiostat (Autolab) had similar peak shapes, peak potentials, and peak currents (Figure 3, Figures S16 and S17). The inset in

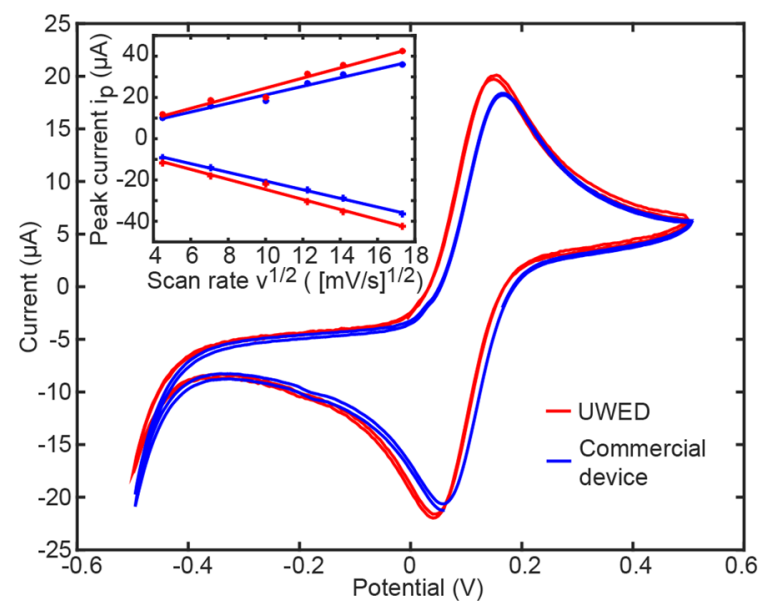

Figure 3. Cyclic voltammogram of $1 \mathrm{mM}$ ferricyanide on a screenprinted electrode at scan rate of $100 \mathrm{mV} / \mathrm{s}$. The inset shows the theoretically expected linear relationship of peak current and square root of scan rate.

Figure 3 demonstrates the theoretically expected linear relationship between the peak current and square root of scan rate for diffusion-controlled processes. ${ }^{40}$ Section 7.3 of the Supporting Information contains all the CVs.

Square-Wave Voltammetry. Square-wave voltammograms (SWVs) of $10 \mu \mathrm{M}-5 \mathrm{mM}$ ferricyanide recorded by the UWED and the commercial potentiostat (AutoLAB) are in good agreement (Figure 4A). Peak amplitudes measured by the UWED were almost uniformly $10 \%$ lower than those measured by the commercial potentiostat. This difference is plausibly due to different timings for sampling of current employed by the UWED and the commercial device for the signal integration. The noise in the SWV measured by the UWED was higher than that of the commercial device, and becomes more notable at low concentrations of ferricyanide. We discuss the noise of the UWED quantitatively in Section 6 of the Supporting Information. This level of noise does not impede analyses over a 100 -fold concentration range. The noise could be further decreased by adjusting the circuit and digital filtering. At high concentrations of ferricyanide $(7.5$ and $10 \mathrm{mM})$, the current exceeded the maximum current range of UWED $( \pm 180 \mu \mathrm{A})$, and resulted in artifacts in the voltammogram. Section 7.4 of the Supporting Information contains all the voltammograms at different concentrations. Figure 4B shows that data obtained by both the UWED and the commercial potentiostat have the theoretically expected linear correlation of peak current and concentration of ferricyanide. Since SWV and differential pulse voltammetry (DPV) have similar voltage sequences and exhibit similar peak shapes, we present only SWV results in this paper. The Supporting Information provides the source code for performing DPV.

\section{CONCLUSIONS}

This paper describes an open-source universal wireless electrochemical detector (UWED). A portable, inexpensive, web-connected electrochemical detector is necessary for successful integration of point-of-care electrochemical diag- 

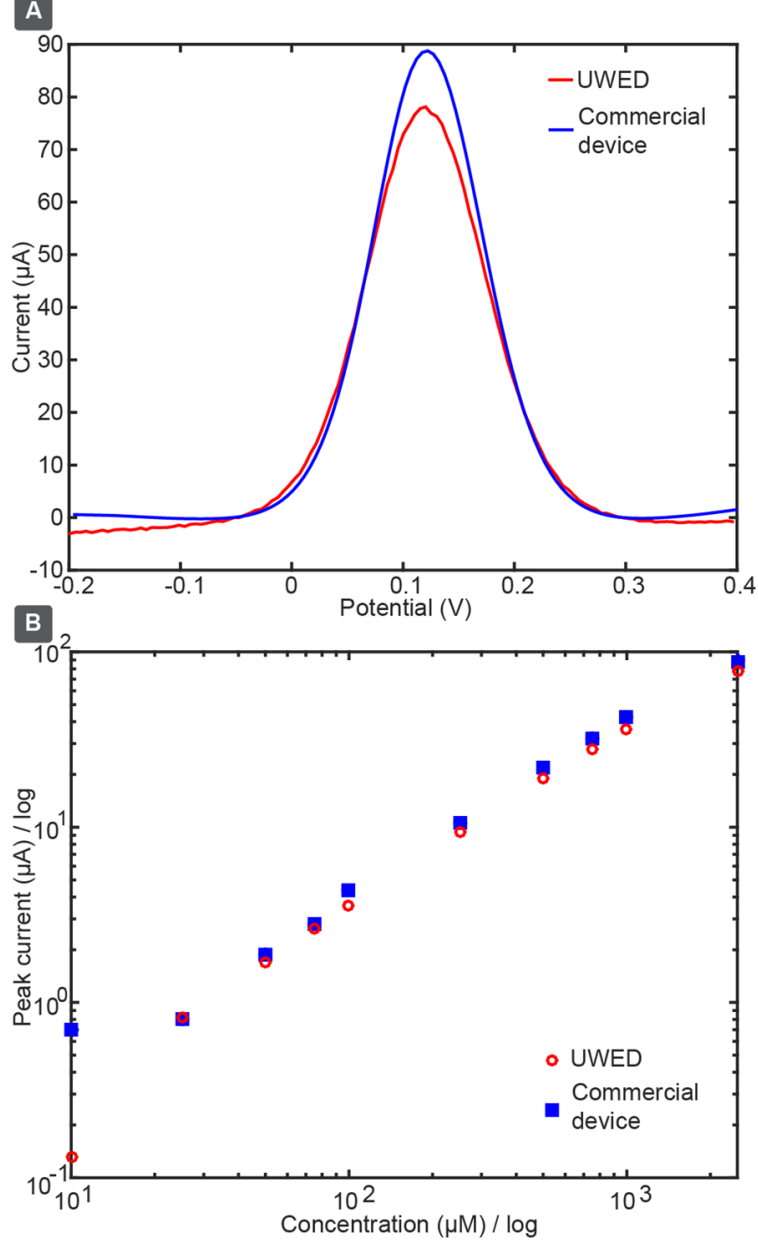

Figure 4. (A) shows the square wave voltammogram recorded in a 1 $\mathrm{mM}$ ferricyanide solution measured by UWED and the commercial potentiostat (step size $5 \mathrm{mV}$, amplitude $50 \mathrm{mV}$, frequency $10 \mathrm{~Hz}$ ). (B) shows the theoretically expected linear correlation between the peak amplitude and concentration of ferricyanide.

nostics for healthcare in the developing world and in resourcelimited settings. The open-source electrochemical detectors provide a valuable resource for development of specialtypurpose electrochemical readers for research, product prototyping, and education. The previously published open-source potentiostats (DStat, ${ }^{18}$ CheapStat, ${ }^{19}$ and a potentiostat/ galvanostat $^{35}$ ) required a wired connection to a computer for operation of the device or data transfer. This work (UWED) presents the design of a wireless electrochemical reader that would be complementary to the prior work. The UWED communicates with a smartphone or a tablet using the wireless Bluetooth Low Energy (BLE) protocol. A host program in the smartphone receives the input parameters of the experiment from the user, communicates the experimental protocol to the UWED, receives the raw data as the result of the experiment from the UWED, visualizes the data for the user, stores the data, and sends the results to the Cloud.

The wireless design and smartphone connectivity of UWED has the following advantages: (i) Since the collection and visualization of data and control of UWED are entirely handled by the smartphone or tablet, we were able to eliminate the elements of the user interface (i.e, buttons and display) from the reader, and adopt a hardware design that is simple, has a small size, and is composed of inexpensive components. (ii)
The UWED allows a physical separation between the electrical reader and the data proxy (e.g., smartphone). This separation minimizes cross-contamination, increases the safety of the user, and enables use of UWED in closed containers (e.g., a glovebox). (iii) The UWED is easily customizable (with no need to make physical changes to its circuit), and the firmware and software of UWED can be updated to accommodate more specialized electrochemical techniques and voltage sequences, such as stripping voltammetry and coulometry. (iv) The Cloud connectivity of smartphones and tablets facilitates the integration of the data in central networks and databases (i.e., in a hospital), and minimizes the errors in manual entering of the results in the database.

We demonstrated application of UWED in the most commonly used electrochemical techniques of potentiometry, chronoamperometry, cyclic voltammetry, and square-wave voltammetry, and showed that the results obtained by UWED are closely comparable to those obtained by a commercial benchtop potentiostat. We have optimized the design of UWED for "do-it-yourself" settings, and chose components that can be manually soldered without specialized equipment such as reflow ovens, and are adaptable to established programming environments. Alternative circuit components can decrease the cost and size of UWED. We hope that the open-source and customizable design of UWED would enable researchers to fabricate a replicate of UWED for their application of interest or integrate the circuit of UWED into different electroanalytical systems.

\section{ASSOCIATED CONTENT}

\section{Supporting Information}

The Supporting Information is available free of charge on the ACS Publications website at DOI: 10.1021/acs.analchem. 8 b00850.

(i) Designs for the electrical circuits and a list of all necessary components, (ii) layout of the printed circuit board, (iii) firmware of the microcontroller, and (iv) code for the software for the smartphone (PDF)

Extended data of electrochemical measurements and electrical characterization of UWED (ZIP)

Video showing device operation (MOV)

\section{AUTHOR INFORMATION}

\section{Corresponding Author}

*E-mail: gwhitesides@gmwgroup.harvard.edu. Phone: +1-617495-9432.

ORCID $\odot$

Jeffrey G. Bell: 0000-0003-3470-1043

M. Teresa Fernández-Abedul: 0000-0003-3782-5025

George M. Whitesides: 0000-0001-9451-2442

\section{Author Contributions}

\#These authors contributed equally.

\section{Notes}

The authors declare no competing financial interest.

\section{ACKNOWLEDGMENTS}

This work was supported by awards from The Bill and Melinda Gates Foundation (award 51308), DTRA (award HDTRA1-14C-0037), and Wyss Institute for Biologically Inspired Engineering. A.A. thanks the Swedish Research Council (VR) for a postdoctoral fellowship. M.N.T. was funded by an International 
Outgoing Marie Curie Fellowship (IOF-GA-2013-622879ENVIRO). J.R. acknowledges the Summer Research Experience for Undergraduates of the Harvard Materials Research Science and Engineering Center (MRSEC REU under NSF award DMR-1420570) program for its support. M.T.F.A. thanks Banco Santander for partially funding her stay through excellence mobility grants (Campus de Excelencia Internacional) for researchers of the University of Oviedo. We thank James MacArthur and the Electronic Instrument Design Lab in Harvard University Department of Physics for providing a productive work environment and useful discussions.

\section{REFERENCES}

(1) Bandodkar, A. J.; Wang, J. Trends Biotechnol. 2014, 32, 363-371.

(2) Windmiller, J. R.; Wang, J. Electroanalysis 2013, 25, 29-46.

(3) Mettakoonpitak, J.; Boehle, K.; Nantaphol, S.; Teengam, P.; Adkins, J. A.; Srisa-Art, M.; Henry, C. S. Electroanalysis 2016, 28, $1420-1436$.

(4) Pedrero, M.; Campuzano, S.; Pingarrón, J. M. Electroanalysis 2014, 26, 1132-1153.

(5) Labib, M.; Sargent, E. H.; Kelley, S. O. Chem. Rev. 2016, 116, 9001-9090.

(6) Duffy, G. F.; Moore, E. J. Anal. Lett. 2017, 50, 1-32.

(7) Wang, J.; Rivas, G.; Cai, X.; Palecek, E.; Nielsen, P.; Shiraishi, H.; Dontha, N.; Luo, D.; Parrado, C.; Chicharro, M.; Farias, P. A. M.; Valera, F. S.; Grant, D. H.; Ozsoz, M.; Flair, M. N. Anal. Chim. Acta 1997, 347, 1-8.

(8) Hanrahan, G.; Patil, D. G.; Wang, J. J. Environ. Monit. 2004, 6, 657-664.

(9) Wang, Y.; Xu, H.; Zhang, J.; Li, G. Sensors 2008, 8, 2043-2081.

(10) Umek, R. M.; Lin, S. W.; Vielmetter, J.; Terbrueggen, R. H.; Irvine, B.; Yu, C. J.; Kayyem, J. F.; Yowanto, H.; Blackburn, G. F.; Farkas, D. H.; Chen, Y.-P. J. Mol. Diagn. 2001, 3, 74-84.

(11) Bandodkar, A. J.; Jia, W.; Wang, J. Electroanalysis 2015, 27, 562572.

(12) Gumpu, M. B.; Sethuraman, S.; Krishnan, U. M.; Rayappan, J. B. B. Sens. Actuators, B 2015, 213, 515-533.

(13) Portable potentiostats, Metrohm, http://www.metrohmautolab.com/Products/Echem/PortablePot/PortablePotentiostats. html, accessed Jan 4, 2018.

(14) Portable potentiostat, BioLogic Science Instruments, http:// www.bio-logic.net/en/products/potentiostat-galvanostat-eis/pg581portable-potentiostatgalvanostat/, accessed Jan 4, 2018.

(15) EmStat, PalmSens Compact Electrochemical Interfaces, https:// www.palmsens.com/product/emstat/, accessed Jan 4, 2018.

(16) Wang, C.-C.; Hennek, J. W.; Ainla, A.; Kumar, A. A.; Lan, W.-J.; Im, J.; Smith, B. S.; Zhao, M.; Whitesides, G. M. Anal. Chem. 2016, 88, 6326-6333.

(17) Nie, Z.; Deiss, F.; Liu, X.; Akbulut, O.; Whitesides, G. M. Lab Chip 2010, 10, 3163-3169.

(18) Dryden, M. D. M.; Wheeler, A. R. PLoS One 2015, 10, e0140349.

(19) Rowe, A. A.; Bonham, A. J.; White, R. J.; Zimmer, M. P.; Yadgar, R. J.; Hobza, T. M.; Honea, J. W.; Ben-Yaacov, I.; Plaxco, K. W. PLoS One 2011, 6, e23783.

(20) Nemiroski, A.; Christodouleas, D. C.; Hennek, J. W.; Kumar, A. A.; Maxwell, E. J.; Fernández-Abedul, M. T.; Whitesides, G. M. Proc. Natl. Acad. Sci. U. S. A. 2014, 111, 11984-11989.

(21) Gao, W.; Emaminejad, S.; Nyein, H. Y. Y.; Challa, S.; Chen, K.; Peck, A.; Fahad, H. M.; Ota, H.; Shiraki, H.; Kiriya, D.; Lien, D.-H.; Brooks, G. A.; Davis, R. W.; Javey, A. Nature 2016, 529, 509-514.

(22) Imani, S.; Bandodkar, A. J.; Mohan, A. M. V.; Kumar, R.; Yu, S.; Wang, J.; Mercier, P. P. Nat. Commun. 2016, 7, 11650.

(23) Panneer Selvam, A.; Muthukumar, S.; Kamakoti, V.; Prasad, S. Sci. Rep. 2016, 6, 23111.

(24) Rose, D. P.; Ratterman, M. E.; Griffin, D. K.; Hou, L.; KelleyLoughnane, N.; Naik, R. R.; Hagen, J. A.; Papautsky, I.; Heikenfeld, J. C. IEEE Trans. Biomed. Eng. 2015, 62, 1457-1465.
(25) Giordano, G. F.; Vicentini, M. B. R.; Murer, R. C.; Augusto, F.; Ferrão, M. F.; Helfer, G. A.; da Costa, A. B.; Gobbi, A. L.; Hantao, L. W.; Lima, R. S. Electrochim. Acta 2016, 219, 170-177.

(26) Sun, A. C.; Yao, C.; Venkatesh, A. G.; Hall, D. A. Sens. Actuators, B 2016, 235, 126-135.

(27) Christidis, K.; Gow, K.; Robertson, P.; Pollard, P. Rev. Sci. Instrum. 2006, 77.

(28) Cruz, A. F. D.; Norena, N.; Kaushik, A.; Bhansali, S. Biosens. Bioelectron. 2014, 62, 249-254.

(29) Hwang, S.; Sonkusale, S. IEEE Sens. J. 2010, 10, 820-821.

(30) Jeanneret, S.; Crespo, G. A.; Ghahraman Afshar, M.; Bakker, E. Sens. Actuators, B 2015, 207, 631-639.

(31) Kappes, T.; Schnierle, P.; Hauser, P. C. Anal. Chim. Acta 1999, $393,77-82$.

(32) Steinberg, M. D.; Lowe, C. R. Sens. Actuators, B 2004, 97, 284289.

(33) Steinberg, M. D.; Kassal, P.; Kereković, I.; Steinberg, I. M. Talanta 2015, 143, 178-183.

(34) Dryden, M. D. M.; Fobel, R.; Fobel, C.; Wheeler, A. R. Anal. Chem. 2017, 89, 4330-4338.

(35) Dobbelaere, T.; Vereecken, P. M.; Detavernier, C. HardwareX 2017, 2, 34-49.

(36) Number of mobile phone users worldwide from 2013 to 2019, Statista, https://www.statista.com/statistics/274774/forecast-ofmobile-phone-users-worldwide/, accessed Jan 4, 2018.

(37) Number of smartphone users worldwide from 2014 to 2020, Statista, https://www.statista.com/statistics/330695/number-ofsmartphone-users-worldwide/, accessed Jan 4, 2018.

(38) Tsaloglou, M.-N.; Nemiroski, A.; Camci-Unal, G.; Christodouleas, D. C.; Murray, L. P.; Connelly, J. T.; Whitesides, G. M. Anal. Biochem. 2018, 543, 116-121.

(39) Buhlmann, P.; Chen, L. D. In Supramolecular Chemistry: From Molecules to Nanomaterials, Steed, J. W., Gale, P. A., Eds.; John Wiley \& Sons Ltd.: Chichester, UK, 2012.

(40) Bard, A. J.; Faulkner, L. R. Electrochemical Methods Fundamentals and Applications, 2nd ed.; John Wiley \& Sons: New York, 2009. 